\title{
Fluid Resuscitation in Shock: Why Starling's Law Is Wrong?
}

\author{
Ahmed N Ghanem* \\ Consultant Urological Surgeon, Mansoura, Egypt \\ *Corresponding author: Ahmed N Ghanem, Consultant Urological Surgeon, Mansoura, Egypt
}

\section{Mini Review}

Professor Fifner's article on "saline versus albumin fluid evaluation (SAFE) 2006 [1], concluding: "saline or albumin produces similar outcome”. In 1998 meta-analysis, albumin fared worse [2], justifying BMJ slogan "Why albumin may not work". This is conflicting and perplexing. Professor Vincent [3] mentioned in his editorial at BMJ: "the aim of the analysis was to show that albumin administration is safe." I wish all the luck with the "Save Albumin Campaign" and confirm that I do not deny albumin safety and usefulness when indicated. The valid painstaking analysis of data and the conclusion of similar or worse outcome, to my mind, re-affirms the fact that albumin oncotic pressure in VIVO is fallacy [3-6], explaining BMJ slogan. It is shameful waste to spend so much effort and money on huge clinical trials, on the wrong basic notion that albumin oncotic pressure exists in VIVO. My aim here, however, is to discuss issues overlooked in all SAFE trials data analysis highlighting a concept that may help to resolve the conflict and more importantly the problems of concerned acutely ill patients on ICU. Fifner mentioned in discussion: "Patients received the amount of fluid the clinician thought necessary to restore or maintain intravascular volume" I applaud the truthful reality of this statement, and believe it pinpoint precisely where the problems are (highlighted here). Thus, if the volume of the given fluid during resuscitation is quantified half the battle is won, and if the scientific basis underlying the thought that mislead physician to infuse such volume is verified and rectified the battle is over. In a letter on the BMJ editorial, I mentioned that SAFE trials and analysis are concerned only with the Type of fluid, albumin versus saline, while missing the important issue of volume, measured in either volumetric or gravimetric method.

Volumetric overload (VO) over Time (VO/T) is a concept verifiable by comparing patients' body weight on ICU to that on hospital admission. This reveals a staggering VO! In 1967, Professor Ashbaugh et al documented fluid gain in the first report on the acute respiratory distress syndrome (ARDS) [7], which became later known as the multiple vital organ dysfunction or failure (MVOD/F) syndrome. It was 1214 litres. Such VO data have not ever since been documented. It is consistently missed in prospective trials. Not a single prospective SAFE or other trial report volumetric data on MVOD/F patients! Fluid Type and Volume, and Time of gain, have vital significance in the pathogenesis and outcome of MVOD/F patients on ICU [8]. Type of fluid gives characteristic serum solute dilution markers. Volume is directly, while time is inversely, related to the severity. Sodiumfree fluids (Type 1) or V01 dilute all serum contents including albumin, but its best marker is hyponatraemia. The well-known transurethral resection of the prostate (TURP) [9] syndrome is a "clean model" of many such hyponatraemia cases seen in clinical practice [1]. A "Clean model" of TURP syndrome means it can be, and has been, precisely reproduced in animals in the absence of sepsis, hypothermia and recognized shocks. Hyponatraemia is common hospital complication of fluid therapy that affects men, women and children and is usually lethal.

The TURP syndrome is induced by both the irrigating fluid absorption (1.5\% Glycine, Sorbitol or Mannitol) and the infused intravenous fluids such as $5 \%$ Glucose [9]. A quantity of 3.5l, gained during 1-hour surgery, induces a classical condition while 5-6l may be lethal. The VO of 3.51 may be considered normal daily intake and is tolerated over couple of hours but when gained in one hour it becomes pathological. The condition manifests clinically with paradoxical hypotension shock (Paradoxical means hyperNOT hypo-volaemic shock) with features unrecognizable from or identical to hypo-volaemic shock except for bradycardia and transient rare elevation of arterial pressure. It also has paradoxical acute renal failure (ARF) among other features of the MVOD/F syndrome. This must to be kept in mind in order to recognize VO/T, induced by SAFE fluids, with scarce markers if any. This is 
important as the TURP procedure is currently performed in saline irrigation (TURIS), so much more VO/T with scarce or no markers will soon appear.

The common thought and practice of treating physician in such paradoxical VO/T shock is to infuse further volume of either SAFE isotonic fluid! He/she aims to elevate pressure by increasing vascular volume in the belief that he is facing hypovolemic hypotension shock, while data indicate VO/T shock. The action just makes it worse or irreversible shock and establishes MVOD/F when the patient is shifted to ICU. The insult of both SAFE isotonic fluids may occur in resuscitating the TURP syndrome with definite characteristic serum markers and proven clinical features, or may complicate overzealous resuscitation of any recognized shock, trauma or ICU patient when serum markers are scarce or nil Nothing to guide physician at all except his thought determined by current basic teaching on vascular volume/ pressures relationship on one hand, and the forces regulating the capillary circulation on the other. The latter determine the type and volume of SAFE fluid used in resuscitation of shock, trauma, burns, hemorrhage and sepsis.

Sodium-based fluids (VO2) such as saline or albumin induce VO/T shock too. It may complicate the resuscitation of the TURP syndrome when VO2 erases hyponatraemia while worsening VO. The main serum marker becomes hypo-albuminaemia [10]. It also has the same clinical features of paradoxical hypotension shock and MVOD/F. It may complicate resuscitation of any recognized shock The transition from hypo- to hyper-volaemic hypotension shock is hard or impossible to detect. No stop signs to show that such patient is having hyper-volaemic not hypo-volaemic hypotension shock. None to warn when the quantity needed in treating true hypovolaemia is surpassed. Vascular pressures of CVP, PCWP and $\mathrm{BP}$ changes of VO/T are identical except for bradicardia and an occasional transient initial rise of BP. Massive plasma and blood infusions have no serum markers or specific vital signs at all except - increase of body weight and MVOD/F. It should be realized that hypotension is not always synonymous with hypovolaemia. It is worth mentioning also that, up to this point, sepsis is as innocent as the wolf in Joseph story. A little later, sepsis will do its nasty work and further complicate MVOD/F into its current trendy name associated with sepsis, termed SIRS. The scientific basis that underlies physician's thought while resuscitating a patient is explained later. The evidence on how and why Starling's law [11] is wrong on all accounts while continuing to dictate the type and incorrect volume of fluid in resuscitation while volume is consistently missed in SAFE trials is explained here.

On the physiological issues; the direct positive relationship of fluid volume and pressure, the work of Poiseuilli on flow and pressure exerted on the wall of strait uniform brass tubes, as well as the albumin oncotic pressure, were all imported by Starling [10] in 1896 direct from physics to medicine at the Lancet without any physiological verification or testing what so ever! Modern clinical chemistry allowed verification of albumin oncotic pressure by Hendry in 1962 [5]. The real ultra-structure of the capillary wall revealed by Karnovesky4 and the pre-capillary sphincter revealed by Rhodin 6 were reported in 1967. The hydrodynamics of a porous orifice tube was reported in 2001 [12]. Thus, all physiological research done before 1962 that advocated Starling's hypothesis and promoted it into law is invalid. Based on the consequences of capillary permeability to macromolecules, Renkin [8] advocated reconsideration of Starling's hypothesis in 1986. What alternative was there then? There was none. Only an idea in mind derived from clinical observation on the use of fluids in resuscitation of shock, trauma and the TURP syndrome was communicated and reported at BMJ [10] in 1985. The direct proportional relationship of fluid volume to pressure works in the vascular system up to a limit only. This is true in physics too, if too much fluid is pushed into a reservoir above its capacity, it will burst, and the volume-pressure relationship vanishes. Thus, perhaps volume replacement in shock should not exceed the maximum capacitance of vascular system of 7 litres in adult. Considering that blood loss is fatal when about half the vascular volume is acutely lost, a replacement should not exceed the lost volume after control of bleeding. After any overzealous vascular volume expansion, the excess must leak out into and drown the interstitial space! The most deleterious effect of such internal drowning is on the vital organs. Both vital organ signs [9] and post-mortem findings [13] demonstrate the massive volume of retained fluids. This letter to Editor [13] is the only documented evidence in literature that reported the massive retained fluid volume with swollen vital organs at postmortem examination! The only article that reported retained fluid volume was the first report on ARDS by Ashbaugh et al. [7].

Albumen oncotic pressure, no doubt, exists in vitro across membrane impermeable to its molecules. Even, in such physics experiments, oncotic pressure is too weak and too slow force to be effectively and solely responsible for fluid return into capillary lumen [5]. It has cell building nutritional value, how does albumin reach the cells? However, the evidence that oncotic pressure works in vivo is non-existing [6]. The only difference between albumen and saline fluids in SAFE trials is the added albumin presumed to have oncotic pressure, a function of its molecule size in relation to pore size or permeability of membrane. The pores of normal capillary wall became known 7 decades after Starling's report and shown to allow horse radish, a much larger molecule than albumin, to pass freely [4]. As the result of this trial1 demonstrated that both SAFE fluids have similar outcome, this further re-affirms that albumen oncotic pressure in clinical medicine [1], clinical chemistry [5] and modern physiology [4,14-16] is fallacy in VIVO, simply because albumen molecules pass freely across the large pores of normal capillary membrane [4]. This may answer the BMJ slogan: Why albumin may not work. So, irrespective whether albumin has equal or worse outcome, the fact that it did not show clear superiority to 
saline in SAFE trials is affirmative evidence that albumen oncotic pressure is fallacy in VIVO. Such fallacy has also been long proved in biochemical [5] and physiological research [4,6]. Oncotic pressure is the presumed main absorption force in capillary-interstitial fluid transfer and represents one half of the equation of Starling's law [11]. Thus, as it has proved wrong, the law must be wrong! This was the reason for repeated calls to reconsider Starling's hypothesis [8]. However, there was no existing alternative then- only an idea in mind communicated at BMJ [10] in 1985. This was later verified and reported, the clinical work [9] in 1990 and physics work [12] in 2001 as well as in more recent articles [17-20].

Verifying the other half of Starling law equation concerning capillary arterial pressure as the filtration force was my objective. Does the capillary have positive pressure on its wall pushing fluid out? Does the flow pressure akin to arterial pressure cause filtration? Does the capillary tube act like Poiseuille' strait uniform tube and have positive pressure on its wall that pushes fluid out through pores? The ultra-structure of capillary wall [4] and precapillary sphincter5 were discovered 70 years after Starling reported his hypothesis. The capillary proved a porous orifice tube. I made several porous tubes fitted with narrow orifice mimicking the capillary with a pre-capillary sphincter on a larger scale to verify this. These porous orifice tubes were used to study hydrodynamic flow and pressure and compared to Poiseuille's tube, particularly in relation to the pressure exerted on its wall. The porous orifice (G) tube dynamics proves totally different to Poiseuille's tube. There was no positive pressure exerted on the wall and no fluid filtered out over the proximal half of the porous orifice tube. The flow pressure representing arterial pressure is not responsible for filtration! It caused mainly suction at the proximal half of the tube. Thus, the main force in the equation on Starling's law concerning arterial capillary pressure filtration is also wrong. How does it work? What pushes the fluid out and what returns it in? Does it offer a complete hypothesis to explain the capillary-interstitial fluid exchange? How does it relate to physiology and medicine? To know the answer to these questions on a most fascinating phenomenon of the $\mathrm{G}$ tube, please read the articles [12,17-20] or the book [21].

So, the law dictating the scientific basis that underlies physician's thought on vascular volume expansion at resuscitation of shock, trauma and acutely ill patients is wrong on all accounts. The most harmful part of this erroneous law is in fact that concerning arterial pressure, presumed to be the main filtration force in the capillary. This is the part that Starling thought acted like Poiseuille's strait uniform tube, exerting positive pressure on the wall that filters fluid out. This underlies the treating physician's thought when embarking on overzealous fluid infusion during the resuscitation of shock. He was taught that volume expansion has direct positive unlimited relationship with pressure. It is the only way he knows off to improve capillary circulation. Well, it does not. Volume replacement is effective when an actual blood volume loss is restored to normal that is less than maximum capacity of the vascular system. After that the relation of volume to pressure is reversed. Any excess volume, vascular expansion or hypervolaemia of VO/T induces hypotension shock just like hypovolaemia does! Considering the concept of VO/T by reporting volume of fluids in future SAFE trials and verifying the scientific basis of fluid resuscitation in shock are needed for resolving the puzzle of MOVD/F and improving outcome of patients on ICU.

\section{References}

1. Finfer $S$ (2006) Effect of baseline serum albumin concentration on outcome of resuscitation with albumin or saline in patients in intensive care units: analysis of data from the saline versus albumin fluid evaluation (SAFE) study. BMJ 333: 1044-1046.

2. Cochrane Injuries Group (1998) Human albumin administration in the critically ill patients: systemic review of randomized controlled trials: Why albumin may not work. BMJ 317: 235-240.

3. Vincent JL (2006) Resuscitation using albumin in critically ill patients: Research in patients at high risk of complications is now needed. BMJ 333: 1029-1030.

4. Karnovesky MJ (1967) The ultra-structural basis of capillary permeability studied with peroxidase as a tracer. J Cell Biol 35: 213-236.

5. Hendry EB (1962) The osmotic pressure and chemical composition of human body fluids. Clinical Chemistry 8(3): 246-265.

6. Rhodin JA (1967) The ultra-structure of mammalian arterioles and precapillary sphincters. J Ultrastructure Research 18: 181-222.

7. Ashbaugh DG, Bigelow DB, Petty TL, Levine BE (1967) Acute respiratory distress in adults. Lancet 2: 319-323.

8. Renkin EM (1986) Some consequences of capillary permeability to macromolecules: Starling's hypothesis reconsidered. Am J Physiol 250(5 Pt 2): 706-710.

9. Ghanem AN, Ward JP (1990) Osmotic and metabolic sequelae of volumetric overload in relation to the TURP syndrome. Br J Urol 66: 7178.

10. Ghanem AN (1985) Hypoalbuminaemic hyponatraemia: a new syndrome? BMJ 291: 1502

11. Starling EH (1886) Factors involved in the causation of dropsy. Lancet 2: 1266-1270, 1330-1334 and 1406-1410.

12. Ghanem AN (2001) Magnetic field-like fluid circulation of a porous orifice tube and relevance to the capillary-interstitial fluid circulation: Preliminary report. Medical Hypotheses 56(3): 325-334.

13. Lessels AM, Honan RP, Haboubi NY, Ali HH, Greene MJ (1982) Death during prostatectomy. J Clin Path 35(1): 117.

14. Reed RK, Rubin K (2010) Transcapillary exchange: role and importance of the interstitial fluid pressure and the extracellular matrix. Cardiovasc Res 87(2): 211-217.

15. Levick JR, Michel CC (2010) Microvascular fluid exchange and the revised Starling principle. Cardiovasc Res 87(2): 198-210.

16. Bulat M, Klarica M (2014) Fluid filtration and reabsorption across microvascular walls: control by oncotic or osmotic pressure? (secondary publication). Croat Med J 55(4): 291-298.

17. Ghanem AN, Ghanem SA (2016) Volumetric Overload Shocks: Why Is Starling's Law for Capillary Interstitial Fluid Transfer Wrong? The Hydrodynamics of a Porous Orifice Tube as Alternative. Surgical Science 7: 245-249.

18. Pindoria N, Ghanem SA, Ghanem KA, Ghanem AN (2017) Volumetric overload shocks in the patho-etiology of the transurethral resection prostatectomy syndrome and acute dilution hyponatraemia. Integr Mol Med. 
19. Ghanem KA, Ghanem AN (2017) The proof and reasons that Starling's law for the capillary- interstitial fluid transfer is wrong, advancing the hydrodynamics of a porous orifice $(\mathrm{G})$ tube as the real mechanism. Blood, Heart and Circ 1(1): 1-7.

20. Ghanem KA, Ghanem AN (2017) Volumetric overload shocks in the patho-etiology of the transurethral resection prostatectomy syndrome and acute dilution hyponatraemia: The clinical evidence based on 23 case series. Basic Research Journal of Medicine and Clinical Sciences $6(4)$.

21. Ghanem AN (2018) Volumetric Overload Shocks. Scholars Press USA. (c) (i) This work is licensed under Creative

To Submit Your Article Click Here: Submit Article

DOI: $10.32474 /$ SCSOAJ.2019.02.000149

$\begin{gathered}\text { Surgery \& Case Studies: Open } \\ \text { Access Journal }\end{gathered}$
Assets of Publishing with us
- Global archiving of articles
- Immediate, unrestricted online access
- Rigorous Peer Review Process
- Authors Retain Copyrights
- Unique DOI for all articles

